\title{
Austenización de aceros con microestructuras diferentes ${ }^{(\cdot)}$
}

\author{
F. García-Caballero*, C. Capdevila*, D. San Martín* y C. García-de Andrés*
}

Resumen Durante los últimos años se han desarrollado algunos modelos físico-matemáticos sobre las transformaciones de fase en enfriamiento continuo e isotérmicas, aplicables a un amplio rango de aceròs. Sin embargo, la modelización de las transformaciones en calentamiento continuo no ha tenido un desarrollo paralelo a las de enfriamiento y su avance ha sido, apreciablemente, menor. En este trabajo se aborda el estudio y modelización de las transformaciones que se producen durante el calentamiento continuo a lo largo de lo que, genéricamente, se denomina la austenización del acero. El principal objetivo es, por consiguiente, describir los procesos que controlan la formación anisotérmica de la austenita en aceros con microestructuras iniciales de ferrita y/o perlita.

Palabras clave Austenización. Modelización. Transformaciones de fase. Cinética. Aceros.

\section{Reaustenitisation of steels with different initial microstructures}

\begin{abstract}
During the last years, physical-mathematical models concerning continuous cooling and isothermal phase transformations valid for a wide range of steels have been attained. However, the modelling of continuous heating transformations has not undergone the same development than in cooling. This investigation concerns with the study and modelisation of continuous heating phase transformations to study the process which is generally referred to as austenitisation of the steel. Therefore, our main aim is to describe those processes which control the non-isothermal formation of austenite in steels with initial microstructures of ferrite and/or pearlite.
\end{abstract}

Keywords Reaustenitisation. Modelling. Phase transformations. Kinetics. Steels.

\section{INTRODUCCIÓN}

El fenómeno de austenización ha sido estudiado en el pasado, solamente a escala superficial y de una forma puntual e inconexa. Para el caso de la formación isotérmica de austenita, a partir de diferentes microestructuras iniciales (puras y mixtas), existen algunos modelos de aproximaciones metalúrgicas específicas ${ }^{[1-5]}$. Sin embargo, ninguno de estos modelos posibilita una aplicación general al calentamiento continuo, excepto, quizás a lentas velocidades de calentamiento que permitan simular condiciones de equilibrio. Por tanto, el objetivo principal de este trabajo es estudiar los mecanismos que controlan la formación anisotérmica de la austenita en aceros con microestructura inicial de ferrita y/o perlita.

\section{MATERIALES Y PROCEDIMIENTO EXPERIMEN- TAL}

La tabla I recoge la composición química de los aceros estudiados. El acero FERR presenta una microestructura completamente ferrítica. En el acero PEARL se llevaron a cabo dos tratamientos isotérmicos diferentes (948 y $798 \mathrm{~K}$ ) para obtener una microestructura completamente perlítica con diferentes parámetros morfológicos. Finalmente, el acero MIXT es un acero bajo en carbono con una microestructura inicial de ferrita y perlita $(87 \%$ de ferrita y $13 \%$ de perlita) como se muestra en la figura 1a).

Existen dos parámetros que caracterizan la morfología de la perlita: el espaciado interlaminar, $\sigma_{\mathrm{o}} \mathrm{y}$ el área por unidad de volumen de intercara de las

(•) Trabajo recibido el día 11 de junio de 2003 y aceptado en su forma final el día 17 de abril de 2004.

$\left(^{*}\right)$ Departamento de Metalurgia Física, Centro Nacional de Investigaciones Metalúrgicas (CENIM), CSIC, Avda. Gregorio del Amo, 8, 28040 Madrid. 
Tabla I. Composición química (\% en masa)

Table I. Chemical composition (mass \%)

\begin{tabular}{cccccccccc}
\hline Aceros & $\mathbf{C}$ & $\mathrm{Mn}$ & $\mathrm{Si}$ & $\mathbf{N}$ & $\mathrm{Al}$ & $\mathbf{P}$ & $\mathbf{C r}$ & $\mathbf{N i}$ & $\mathbf{N b}$ \\
\hline FERR & 0,002 & 0,05 & - & 0,004 & - & 0,003 & - & - & - \\
PEARL & 0,76 & 0,91 & 0,24 & - & - & 0,013 & - & - & - \\
MIXT & 0,11 & 1,47 & 0,27 & 0,005 & 0,039 & 0,015 & 0,03 & 0,03 & 0,03 \\
\hline
\end{tabular}
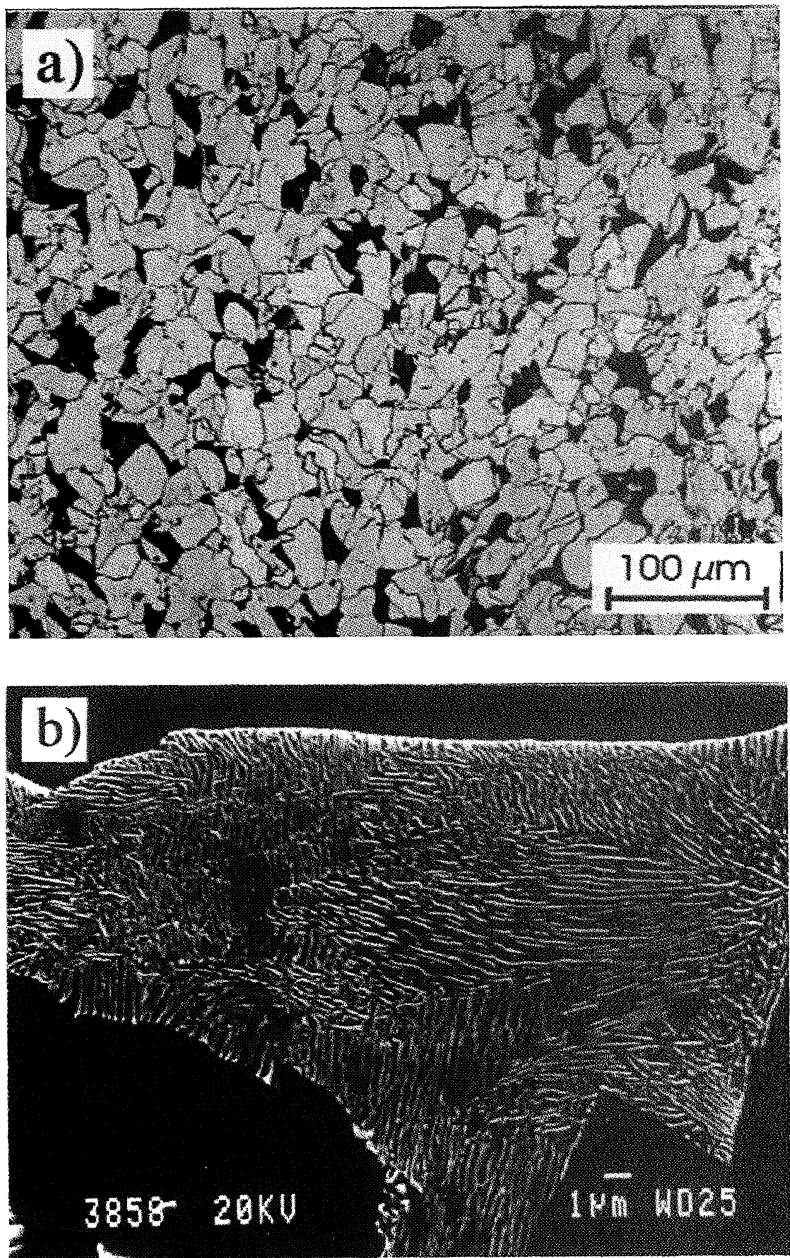

Figura 1. Microestructura inicial del acero MIXT: (a) micrografía óptica; (b) Micrografía electrónica de barrido de un nódulo de perlita.

Figure 1. Initial microstructure of MIXT steel: (a) Optical micrograph; (b) Scanning electron micrograph of pearlite.

colonias perlíticas, $S_{v}^{P P[4]}$. Aproximando la colonia perlítica a un octaedro truncado, la longitud de borde de las colonias perlíticas, $a^{P}$, se puede calcular a partir de $S_{v}^{P P}$. Los valores de $\sigma_{0}, S_{v}^{P P}$ y $a^{P}$ para el acero MIXT se encuentran recogidos en la tabla II. Así mismo, los resultados de caracterización morfológica de la perlita en el acero PEARL fueron publicados en otro trabajo ${ }^{[6]}$, en el que aparece
Tabla II. Caracterización morfológica de la perlita en el acero MIXT

Table II. Morphological characterisation of pearlite in MIXT steel

\begin{tabular}{cccc}
\hline Acero & $\sigma_{0} \times 10^{-3}, \mathrm{~mm}$ & $\mathrm{~S}^{\mathrm{PP}}, \mathrm{mm}^{-1}$ & $a^{\mathrm{P}} \times 10^{-3}, \mathrm{~mm}$ \\
\hline MIXT & $0,15 \pm 0,02$ & $1.289 \pm 323$ & $1,03 \pm 0,35$ \\
\hline
\end{tabular}

también publicado el diámetro de grano ferrítico medio $(D)$ medido en el acero FERR.

Para la validación experimental de los modelos de austenización se utilizó un dilatómetro de alta resolución ADAMEL LHOMARGY DT1000. A partir de las curvas dilatometrícas de calentamiento se seleccionaron diferentes temperaturas de temple con el fin de investigar el progreso de la transformación, el cual se determina a través de la evolución de la fracción de volumen de martensita en muestras atacadas con reactivo LePera (Fig. 2).

\section{RESULTADOS Y DISCUSIÓN}

\subsection{Modelización de las cinéticas de formación anisotérmica de austenita en un acero con microestructura inicial ferrítica}

En la formación de austenita a partir de la ferrita, el crecimiento de la austenita está controlado por procesos en la intercara y la velocidad de crecimiento $G$ viene dada por:

$$
\begin{gathered}
\mathrm{G}=\frac{\delta v}{k T} \exp \left(-\frac{\Delta \mathrm{G}_{a c t}}{k T}\right) \Delta \mathrm{g}^{\alpha \rightarrow \gamma}= \\
=\frac{\delta v}{k T} \exp \left(\frac{\Delta S}{k}\right) \exp \left(-\frac{\Delta H}{k T}\right) \Delta \mathrm{g}^{\alpha \rightarrow \gamma}\{T\}
\end{gathered}
$$

donde, $\delta$ es el espesor de la intercara, v es el número de intentos para saltar la barrera energética de la frontera por unidad de tiempo, $k$ es la constante de Bolztman, $T$ es la temperatura absoluta, $\Delta \mathrm{G}_{\text {act }}$ es la energía libre para la transferencia de átomos a 

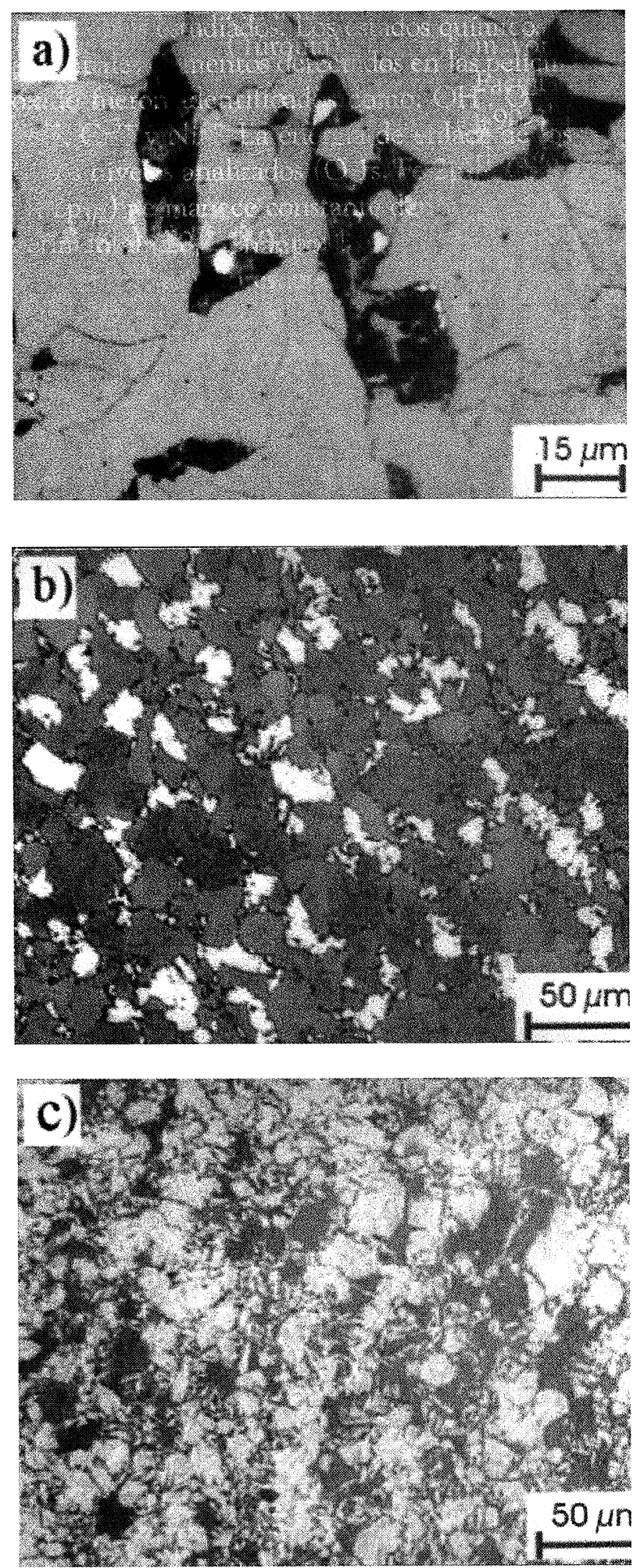

Figura 2. Micrografías ópticas de diferentes estadios del proceso de austenización del acero MIXT. (a) $1017 \mathrm{~K}$; (b) $1084 \mathrm{~K}$; (c) $1135 \mathrm{~K}$; Velocidad de calentamiento $5 \mathrm{~K} / \mathrm{s}$.

Figure 2. Optical micrographs at different stages of the reaustenitisation process in MIXT steel. (a) $1017 \mathrm{~K}$; (b) 1084 $\mathrm{K}$; (c) $1135 \mathrm{~K}$; Heating rate of $5 \mathrm{~K} / \mathrm{s}$.

través de la intercara $\alpha / \gamma, \Delta S$ es la entropía de activación por átomo, $\Delta H$ es la entalpía de activación por átomo, y $\Delta \mathrm{g}^{\alpha \rightarrow \gamma}$ es la diferencia de energía libre de Gibbs por átomo entre las fases $\alpha$ y $\gamma^{[7]}$. Suponiendo que la saturación de lugares de nucleación tiene lugar y que la reacción viene controlada por el crecimiento, las leyes cinéticas obtenidas para los tres lugares activados de crecimiento son las siguientes:

$$
V_{\gamma}=1-\exp \left[-\left(K_{s} t+K_{e} t^{2}+K_{c} t^{3}\right)\right]
$$

donde, $V_{\gamma}$ representa la fracción de volumen de austenita formada, $t$ es el tiempo y $K_{s}, K_{e} y$ $K_{c}$ vienen dados por:

$$
K_{s}=2 \mathrm{G} \frac{3,35}{D} ; K_{e}=\pi \mathrm{G}^{2} \frac{8,5}{D^{2}} ; K_{c}=\frac{4}{3} \pi \mathrm{G}^{3} \frac{12}{D^{3}}
$$

Puesto que la condición de Avrami para una reacción isocinética no se satisface en calentamiento continuo, se obtuvo la siguiente ecuación general para el cálculo de la fracción de volumen de austenita $\left(V_{\gamma}\right)$ presente en la microestructura como una función de la temperatura ${ }^{[7]}$ :

$$
\begin{gathered}
V_{\gamma}=1-\exp \left\{-\int_{T_{s}}^{T}\left[\frac{6,7}{\dot{T} D} G+\frac{53,4}{(\dot{T} D)^{2}} G^{2}\left(T-T_{s}\right)+\right.\right. \\
\left.\left.+\frac{150,8}{(\dot{T} D)^{3}} G^{3}\left(T-T_{s}\right)^{2}\right] d T\right\}
\end{gathered}
$$

donde, $T_{s}$ es la temperatura de inicio de la trasformación.

La transformación $\alpha \rightarrow \gamma$ en el calentamiento continuo es una transformación tan rápida que no es posible medir experimentalmente fracciones de volumen de austenita en estados intermedios de la transformación. Por eso, la validación de este modelo se ha realizado mediante la comparación de curvas dilatométricas de calentamiento experimentales y teóricas ${ }^{[7]}$. Los resultados de esta validación en el acero FERR muestran un excelente acuerdo entre los resultados predichos por el modelo y los experimentales.

\subsection{Modelización de las cinéticas de formación anisotérmica de austenita en un acero con microestructura inicial perlítica}

En general, los procesos de nucleación y crecimiento bajo condiciones isotérmicas pueden ser descritos utilizando la ecuación de Avrami: 


$$
V_{\gamma}=1-\exp \left(-\frac{\pi}{3} \dot{N} G^{3} t^{4}\right)
$$

donde $V_{\gamma}$ representa la fracción de volumen de austenita formada, $t$ el tiempo, $\dot{N}$ la velocidad de nucleación y $G$ la velocidad de crecimiento. Expresiones para el cálculo de $\dot{N}$ y G en función de la temperatura y de la microestructura fueron propuestas en otro trabajo ${ }^{[8]}$.

Como para la transformación de ferrita a austenita, en este caso se ha obtenido también la siguiente ecuación general para describir la transformación anisotérmica de perlita a austenita en un acero perlítico ${ }^{[8]}$ :

$$
V_{\gamma}=1-\exp \left(-\int_{A C_{1}}^{T} \frac{4 \pi}{3(\dot{T})^{2}} \dot{N} G^{3} \Delta T^{3} d T\right)
$$

La temperatura eutectoide $A c_{1}$ del acero se determinó utilizando la fórmula de Andrews ${ }^{[9]}$. La validación experimental de este modelo fue publicada en otro trabajo ${ }^{[8]}$ y demostró que la precisión y fiabilidad de este modelo es superior al $90 \%$.

\subsection{Modelización de las cinéticas de formación no isotérmica de austenita en un acero con microestructura inicial de ferrita más perlita}

En la austenización de microestructuras compuestas por ferrita y perlita tienen lugar dos transformaciones diferentes: la disolución de la perlita y la transformación de la ferrita en austenita. Los procesos de nucleación y crecimiento que controlan la disolución de perlita en un acero con dicha microestructura son los mismos que los descritos anteriormente para un acero perlítico. Por lo tanto, la fracción de volumen de austenita obtenida a partir de la disolución de la perlita, $V_{\gamma}^{\mathrm{P}}$, vendrá dada por la siguiente expresión:

$$
V_{\gamma}^{P}=V_{P_{0}}\left\{1-\exp \left[-\int_{A C_{1}}^{T} \frac{4 \pi}{3(\dot{T})^{4}} \dot{N} G^{3} \Delta T^{3} d T\right]\right\}
$$

donde $V_{P_{0}}$ es la fracción de volumen de perlita presente en la microestructura inicial.

Una vez disuelta la perlita, la austenita procedente de la disolución de la perlita y, por tanto, enriquecida en carbono $(0,8 \%$ en masa), crecerá a expensas de la ferrita. El modelo desarrollado para esta segunda parte de la austenización incluye las siguientes consideraciones:
- no hay formación de nuevos núcleos de austenita durante la transformación $\alpha \rightarrow \gamma$

- se considera una geometría plana en el movimiento de la intercara $\gamma / \alpha$

- la curva de calentamiento se puede describir como una sucesión de pequeños intervalos isotérmicos, $d t$

- se supone equilibrio local de la austenita

- finalmente, cada intervalo, $d t$, va a ser tan pequeño como para poder considerar que el gradiente de concentración de carbono en el interior de la austenita se mantiene invariante con el tiempo a cada temperatura.

El proceso de austenización va a estar controlado por la difusión de carbono en el interior de la austenita. Durante el calentamiento, los átomos de carbono van a difundir desde el interior de los granos de austenita hasta la intercara $\gamma / \alpha$, para pasar a la ferrita. De esta forma, la intercara se mueve, transformando parte de la ferrita en austenita. Este proceso continúa hasta que la composición promedio de carbono en la austenita sea igual al contenido nominal del acero, momento en el que toda la ferrita se habrá transformado (temperatura $A c_{3}$ ) y la microestructura será completamente austenítica.

En este trabajo se considera que los granos de austenita son esferas cuyo radio varía de acuerdo con la expresión $r^{\gamma \alpha}=\eta t^{1 / 2}$, siendo $\eta$ la constante de crecimiento parabólico de la transformación; en este caso,

$$
\eta=\left[D_{C}^{\gamma} \frac{\left(C^{C}-C^{\gamma \alpha}\right)\left(2 C^{e u t}-C^{C}-C^{\gamma \alpha}\right)}{\left(C^{\gamma \alpha}-C^{\alpha \gamma}\right)\left(C^{e u t}-C^{\alpha \gamma}\right)}\right]^{1 / 2}
$$

con $C^{c}, C^{\gamma \alpha}, C^{\alpha \gamma}$ y $C^{e u t}$, la concentración de carbono en el centro de los granos austeníticos, en la interfase $\gamma / \alpha$ dentro de la austenita y dentro de la ferrita, y la concentración del punto eutectoide $(0,8 \%$ C), respectivamente. Si se tiene en cuenta la teoría de Avrami $^{[10]}$ que relaciona el volumen extendido con el volumen real transformado y, considerando que la transformación tiene lugar durante un calentamiento continuo, tal que $d t=d T / \dot{T}$, la evolución de la fracción de volumen de austenita durante la transformación $\alpha \rightarrow \gamma$ se puede expresar de la forma,

$$
V_{\gamma}^{\alpha}=V_{\alpha_{o}}\left\{1-\exp \left[-\frac{\left[6 \pi^{2}\left(N_{V}^{o}\right)^{2}\right]^{1 / 2}}{\dot{T}^{3 / 2}}\left(\frac{2}{3} \int_{A c_{p}}^{T} \eta^{2} d T\right)^{3 / 2}\right]\right\}
$$


donde, $V_{\gamma}^{\alpha}, V_{\alpha_{0}}, A c_{p}$ y $N_{V}^{o}$ son la fracción de volumen de austenita formada a partir de la ferrita, la fracción de volumen inicial de la ferrita, la temperatura a la que toda la perlita se ha disuelto, y el número de granos de austenita por unidad de volumen de ferrita al final de la disolución de la perlita, respectivamente. $N_{V}^{o}$ viene dado por,

$$
N_{V}^{0}=\frac{V_{P_{0}}}{V_{\alpha_{0}}} \frac{1}{\dot{T}} \int_{A C_{1}}^{T} \dot{N}\left(V_{P_{0}}-V_{\gamma}^{P}\right) d T
$$

La figura 3 muestra las predicciones teóricas de la evolución de la fracción de volumen de austenita, obtenidas a partir de la ecuación (8), junto con los resultados experimentales para una velocidad de calentamiento de $5 \mathrm{~K} / \mathrm{s}$. Se puede concluir que el modelo presentado predice de una manera muy precisa la evolución de la fracción de volumen de austenita.

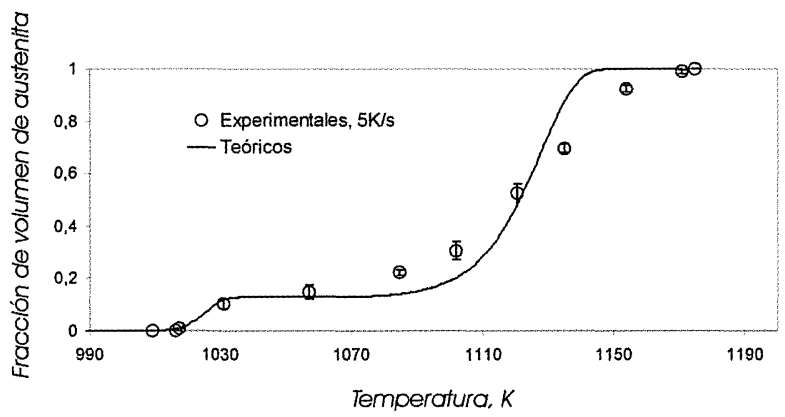

Figura 3. Resultados cinéticos teóricos y experimentales de la formación de austenita en calentamiento continuo en el acero MIXT.

Figure 3. Experimental and calculated kinetics results for the formation of austenite under continuous heating conditions in MIXT steel.

\section{CONCLUSIONES}

Se ha utilizado el conocimiento teórico acerca de la formación isotérmica de austenita, a partir de microestructuras iniciales puras y mixtas para desarrollar un modelo para la formación anisotérmica de austenita en aceros con microestructura inicial formada por ferrita y/o perlita. Se usó la ecuación de Avrami para reproducir la cinética de formación de austenita durante el calentamiento continuo.

\section{Agradecimientos}

Los autores agradecen la ayuda económica del $\mathrm{Mi}$ nisterio de Ciencia y Tecnología (MAT20011617). F.G. Caballero agradece al mismo Ministerio su ayuda en forma de contrato Ramón y Cajal (Programa RyC 2002).

\section{REFERENCIAS}

[1] R.R. Judd y H.W. Paxton, Trans. Tms-Aime 242 (1968) 206-215.

[2] M. Hillert, K. NilsSON y L.E. TORNDAHL, JISI 209 (1971) 49-66.

[3] G.R. Speich, V.A. Demarest y R.L. Miller, Met. Trans. A 12A (1981) 1419-1428.

[4] A. Roosz, Z. Gacsi y E.G. Fuchs, Acta Metall. 31 (1983) 509-517.

[5] R. MAncini and C. Budde, Rev. Metal. Madrid 38 (2002) 426-432.

[6] F.G. Caballero, C. Capdevila y C. García de Andrés, ISIJ International 41 (2001) 1093-1102.

[7] F.G. Caballero, C. Capdevila y C. García de Andrés, Mater. Sci. Tech. 17 (2001) 1114-1118.

[8] F.G. Caballero, C. Capdevila y C. García de Andrés, Met. Trans. A 32A (2001) 1283-1291.

[9] K.W. ANDREWS, JISI 203 (1965) 721-725.

[10] M. Avrami, J. Chem. Phys. 8 (1940) 212-224. 